\title{
Fatores associados à prática de atividade física no lazer: análise dos brasileiros com doenças crônicas
}

\author{
Factors associated with leisure-time physical activity: \\ analysis of Brazilians with chronic diseases
}

Paulo Sergio Cardoso da Silva (https://orcid.org/0000-0002-7271-2782) ${ }^{1}$

Antonio Fernando Boing (https://orcid.org/0000-0001-9331-1550) ${ }^{1}$

${ }^{1}$ Programa de PósGraduação em Saúde Coletiva, Departamento de Saúde Pública, Universidade Federal de Santa Catarina. R. Eng. Agronômico Andrei Cristian Ferreira s/n, Trindade. 88040-900 Florianópolis SC Brasil. pauloscs85@gmail.com
Abstract The scope of this study was to analy$z e$ the prevalence and the factors associated with sufficient leisure-time physical activity (LTPA) among Brazilians diagnosed with hypertension, diabetes and/or hypercholesterolemia. It involved a representative, cross-sectional population-based study for the entire Brazilian territory. Adults aged 20 years and over were interviewed. Prevalences were estimated and, by means of logistic regression, the crude and adjusted odds ratios (OR) of the outcome were calculated in accordance with each of the exploratory variables. The prevalence of sufficient LTPA was 15.9\%, 15.3\% and $21.4 \%$ among people with hypertension $(n=12,228)$, diabetes $(n=3,577)$ and hypercholesterolemia $(n=7,124)$, respectively. These values were lower than those observed among adults who did not report one of the three diseases. Adjusted analysis showed that individuals with positive self-perception of health and with more schooling, who reported a public space near their homes for physical activity, the existence in the city of public programs that encourage physical activity and the recommendation of health professionals to practice LTPA, were associated with a greater chance of being sufficiently active.

Key words Physical activity, Non-communicable chronic diseases, Health surveys, Epidemiology, Brazil
Resumo $O$ objetivo desse estudo foi analisar a prevalência e os fatores associados à prática de atividade física no lazer (PAFL) suficiente em brasileiros com diagnósticos de hipertensão arterial, diabetes e/ou hipercolesterolemia. Trata-se de um estudo transversal de base populacional com representatividade para todo o território brasileiro. Foram entrevistados adultos com 20 ou mais anos de idade. Foram estimadas prevalências e, por meio de regressão logística, calculadas as razões de chance (OR) bruta e ajustada do desfecho segundo cada uma das variáveis exploratórias. A prevalência de PAFL suficiente foi de 15,9\%, 15,3\% e 21,4\% entre as pessoas com hipertensão arterial $(n=12.228)$, diabetes $(n=3.577)$ e hipercolesterolemia $(n=7.124)$, respectivamente. Tais valores foram inferiores ao observado entre os adultos que não referiram uma das três doenças. Análise ajustada mostrou que indivíduos com autopercepção positiva da saúde, com maior escolaridade, que referiram espaço público próximo ao domicílio para a realização de atividade física, presença no município de programas públicos que estimulem a prática de atividade física e recomendação de profissional de saúde para a PAFL estiveram associados a maior chance de serem suficientemente ativos. Palavras-chave Atividade física, Doenças crônicas não transmissíveis, Inquéritos epidemiológicos, Epidemiologia, Brasil 


\section{Introdução}

O controle das doenças crônicas não transmissíveis (DCNT) é um dos maiores desafios do setor saúde no início do século XXI. Em conjunto, as doenças cardiovasculares, o diabetes, os cânceres e as doenças pulmonares são responsáveis por matar 41 milhões de pessoas por ano em todo o mundo, o que corresponde a $71 \%$ de todos os óbitos ${ }^{1}$. No Brasil, estudos apontam que $45 \%$ da população com 18 anos ou mais relatou ter pelo menos uma DCNT em 2013, valor que correspondia a mais de 66 milhões de pessoas ${ }^{2}$. Entre as principais DCNT estão a hipertensão arterial (HAS), o diabetes e a hipercolesterolemia ${ }^{3,4}$, com prevalências, em adultos e idosos, de 40,7\%, 7,1\% e $14,7 \%$, respectivamente 3 .

Diante desse quadro epidemiológico, o Governo Federal criou em 2011 o Plano de Ações Estratégicas para o Enfrentamento das DCNT no Brasil 2011-2022, que contempla o comportamento saudável como um dos seus eixos principais $^{5}$. Considerada como um dos principais fatores associados às DCNT, a prática regular de atividade física traz inúmeros benefícios à saúde, sendo fator de proteção para diferentes doenças e também forma de tratamento não medicamentoso entre aqueles que já apresentam alguma patologia ${ }^{3,6-13}$.

A prática regular de atividade física é capaz de reduzir o risco de mortalidade ${ }^{6,7}$, melhorar a pressão arterial ${ }^{9,14}$, melhorar o controle glicêmico, peso corporal, marcadores de inflamação e de risco cardiovascular ${ }^{12,13,15}$, reduzir as taxas de colesterol da lipoproteína de baixa densidade (LDL-C), elevar o colesterol da lipoproteína de alta densidade (HDL-C) e elevar a sensibilidade à insulina ${ }^{10}$.

Ainda que com reconhecidos benefícios à saúde, estima-se que mais de 1,4 bilhão de adultos em todo o mundo não sejam suficientemente ativos $^{16}$. No Brasil, em 2013, apenas 22,5\% dos brasileiros eram suficientemente ativos no lazer $^{17,18}$. Quando considerada a população das capitais brasileiras, dados do Ministério da Saúde apontaram prevalência de $37,6 \%$ para prática suficiente de atividade física nos adultos e idosos ${ }^{19}$. Entre adultos brasileiros com dislipidemia, HAS ou diabetes, estudo apontou prevalência de prática de atividade física (PAF) no lazer ainda menor que a média populacional ${ }^{20}$.

Na população em geral características individuais como ser mais jovem, do sexo masculino, ter maior escolaridade e renda e ter melhor percepção da saúde estão associados a uma maior
PAF $^{17-19,21-24}$. Também associado a maiores níveis de PAF está o ambiente em que as pessoasvivem, como o acesso a praças, ruas com alta conectividade e seguras, e o acesso aos serviços de saú$\mathrm{de}^{21,25-28}$.

Contudo, mesmo consolidada na literatura a associação inversa entre a prática de atividade física e a ocorrência de DCNT ${ }^{3,6,7,9}$, poucos estudos analisam sua distribuição e fatores associados em pessoas já com DCNT ${ }^{13,20,29}$, sendo eles geralmente baseados em amostras locorregionais e analisando exclusivamente características individuais e não incorporando variáveis contextuais sobre $o$ ambiente em que as pessoas vivem $22,26,27,29$.

A alta ocorrência das DCNT no Brasil ${ }^{2,4}$, somada à baixa prevalência de PAF suficiente nesta população ${ }^{9,20,30}$ e aos elevados custos em saúde pública relacionados a este contexto ${ }^{31}$, constituem um cenário bastante preocupante. Neste sentido, ampliar o conhecimento sobre a prática de atividade física entre pessoas com doença crônica é essencial para a vigilância em saúde e o desenho de políticas e ações.

O objetivo desse estudo foi analisar a prática de atividade física no lazer (PAFL) em brasileiros adultos com HAS, diabetes e hipercolesterolemia e seus fatores associados.

\section{Métodos}

O presente estudo analisou dados provenientes da PNS de 2013, realizada pelo Instituto Brasileiro de Geografia e Estatística (IBGE). A PNS é uma pesquisa de caráter domiciliar, com representatividade nacional e base populacional realizada em amostragem de três estágios: os setores censitários ou conjunto de setores formaram as unidades primárias de amostragem; os domicílios formam as unidades do segundo estágio; e os moradores de 18 anos ou mais de idade as unidades de terceiro estágio. A PNS 2013 realizou entrevistas em 64.348 domicílios tendo 60.202 adultos respondido ao questionário individual com informações sobre o estado de saúde, estilo de vida e doenças crônicas. Mais detalhes metodológicos da PNS podem ser encontrados em publicação própria da pesquisa ${ }^{32}$.

A variável de desfecho foi a prática de atividade física no lazer. Ela teve sua categorização dicotomizada em prática suficiente ou não de atividade física no lazer. Para identificar os indivíduos suficientemente ativos no lazer, considerou-se a realização de pelo menos 150 minutos/ semana de atividades físicas leves ou moderadas, 
ou pelo menos 75 minutos/semana de atividades físicas vigorosas no lazer, ou um combinado de atividades físicas moderadas e vigorosas totalizando 150 minutos semanais ${ }^{33}$. Sua construção se deu a partir do questionamento se o entrevistado havia praticado algum tipo de exercício físico ou esporte nos últimos três meses. Aos que responderam positivamente, foi então indagado quantos dias por semana a pessoa costumava praticar exercício físico ou esporte e quantas horas e minutos em geral durava a atividade. Foram consideradas como atividade física leve ou moderada a caminhada, musculação, hidroginástica, ginástica geral/localizada/pilates/alongamento, natação, artes marciais e luta, bicicleta/ergométrica, voleibol e dança. Como atividade física vigorosa estavam previstas corrida, ginástica aeróbica/spinning/step/ jump, futebol, basquetebol e tênis. Por atividade física considerou-se qualquer movimento corporal produzido pela contração do músculo esquelético que aumenta o gasto de energia acima do nível basal ou de repouso ${ }^{34}$, sendo o domínio lazer aquele realizado a critério de quem não está trabalhando, transportando para um local diferente e não realizando tarefas domésticas, sendo exemplo os esportes ou exercícios, caminhadas e jogos ${ }^{35}$.

Já as variáveis exploratórias foram sexo (masculino; feminino), raça/cor de pele (preta, parda e branca; pessoas que autorreferiram cor/raça indígena e amarela foram excluídas da análise devido ao baixo número de entrevistados), idade (20 a 39 anos; 40 a 59 e 60 ou mais),estado civil (casado; separado/divorciado ou viúvo; solteiro), escolaridade (sem instrução, ensino fundamental completo/incompleto, ensino médio incompleto/completo e ensino superior incompleto/completo), autopercepção do estado de saúde (boa - que considerou as respostas boa e muito boa; ruim - que considerou as respostas regular, ruim e muito ruim), autorrelato de presença de espaço público perto do domicílio para a realização de atividade física ( $\operatorname{sim}$; não); autorrelato da presença no município de programas públicos que estimulem a prática de atividade física ( $\operatorname{sim}$; não); e recomendação de profissional de saúde para a PAF (sim; não).

As análises foram estratificadas de acordo com a presença da patologia investigada, HAS $(n=12.288)$, diabetes $(n=3.577)$ e hipercolesterolemia $(n=7.124)$. Para todas as pessoas indagava-se: "Algum médico já lhe deu o diagnóstico de < patologia >?", com resposta dicotômica "sim" e "não". Assim, os dados foram analisados separadamente entre os que não apresentavam nenhuma dessas doenças e entre os que referiram diagnóstico de cada uma delas. Para a análise da PAFL suficiente foram testadas as associações do desfecho com as demais variáveis, sendo estimadas as prevalências por categoria e por meio de regressão logística as razões de chance (OR) brutas e ajustadas com seus respectivos intervalos de confiança de $95 \%$.

Os dados foram analisados no programa Stata versão 12.0, sendo considerados os pesos amostrais e efeitos da amostragem complexa. A PNS teve aprovação da Comissão Nacional de Ética em Pesquisa (CONEP). Por ser gerida pelo IBGE e ter domínio público, os dados estão disponíveis sem identificação dos entrevistados. Desta forma, não se fez necessária nova submissão a Comitê de Ética em Pesquisa.

\section{Resultados}

A prevalência de PAFL suficiente em adultos com 20 ou mais anos de idade foi de 15,9\%, 15,3\% e $21,4 \%$ entre as pessoas com HAS, diabetes e hipercolesterolemia, respectivamente. Naqueles indivíduos sem nenhuma das três doenças, a prevalência foi de 22,7\% (Tabela 1). Entre os que relataram alguma das três patologias investigadas, verificou-se maior prevalência de PAF suficientenos mais jovens, que se autodeclararam brancos, com maior escolaridade, com autopercepção positiva da saúde, que autorrelataram a presença de espaço público para a PAF perto do domicílio, de programas públicos que estimulem a PAF no município e que receberam recomendação de profissional de saúde para a PAF (Tabela 1).

Entre os indivíduos sem nenhuma das três patologias, análise bruta apontou maior chance de PAF suficiente entre homens, jovens, de cor branca, solteiros, com maior escolaridade, com percepção positiva da saúde, e entre aqueles que relataram a presença de espaços e programas públicos para a prática de atividade física (Tabela 2 ).

Ainda quando avaliados indivíduos sem nenhuma das patologias investigadas, modelo ajustado de regressão logística mostrou maior chance de PAFL suficiente entre os homens (duas vezes maior) comparados às mulheres; entre os mais jovens ( $81 \%$ maior) comparados aos mais idosos; e entre aqueles com maior escolaridade (4,5 vezes maior) comparados aos sem instrução. Ter autopercepção positiva da saúde, presença de espaço público e de programas públicos para estimular a prática de atividade física aumentaram as chances de PAFL suficiente em 48\%, 66\% e $49 \%$, respectivamente (Tabela 2 ). 


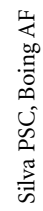

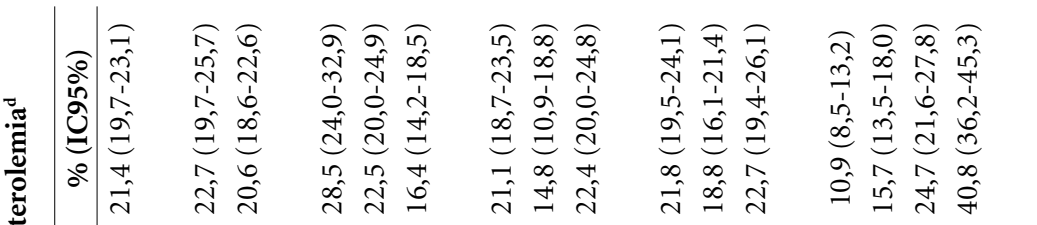

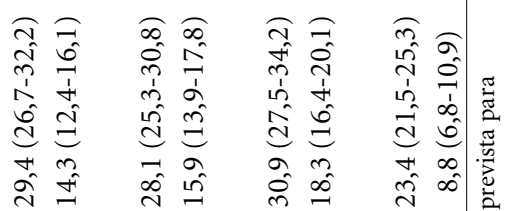

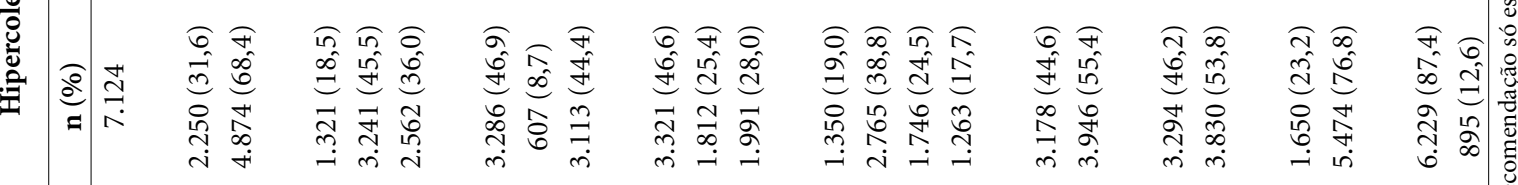

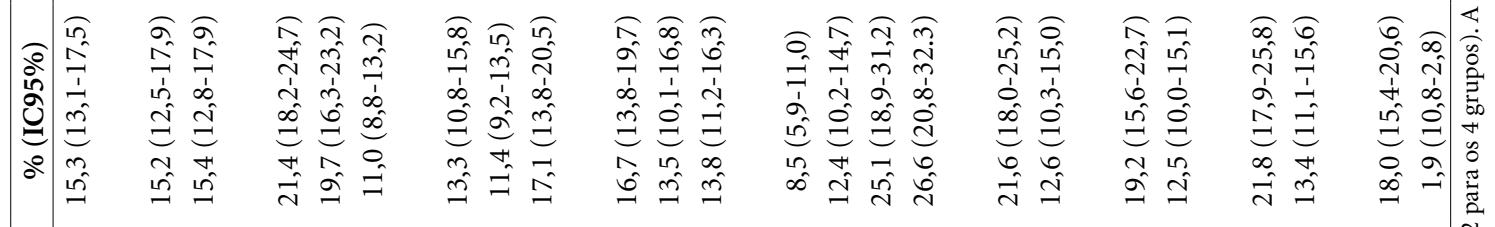

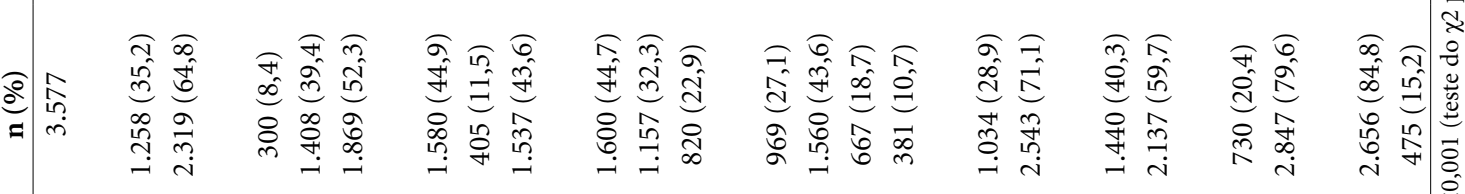

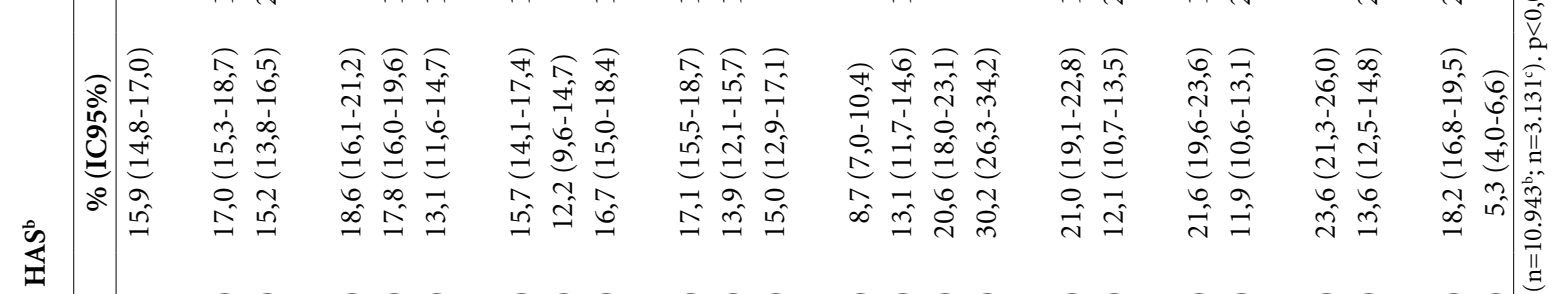

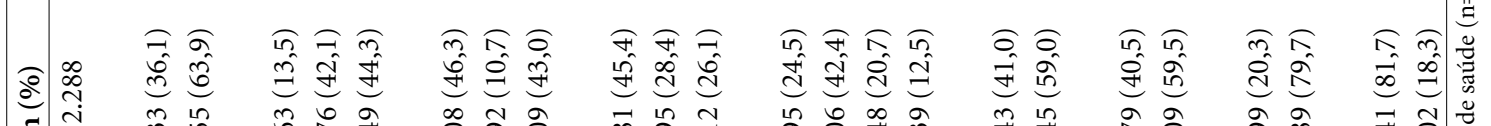

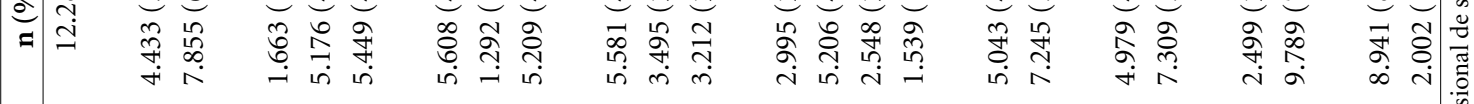




Tabela 2. Razão de chance bruta e ajustada para PAFL suficiente em adultos ( $\geq 20$ anos) segundo presença de hipertensão arterial (HAS) e fatores associados. PNS 2013.

\begin{tabular}{|c|c|c|c|c|c|c|c|c|}
\hline \multirow{3}{*}{ Variáveis } & \multicolumn{4}{|c|}{$\begin{array}{l}\text { Sem HAS, diabetes e } \\
\text { hipercolesterolemia }\end{array}$} & \multicolumn{4}{|c|}{ Com HAS } \\
\hline & \multicolumn{2}{|r|}{ Bruta } & \multicolumn{2}{|c|}{ Ajustada } & \multicolumn{2}{|r|}{ Bruta } & \multicolumn{2}{|c|}{ Ajustada } \\
\hline & OR & IC & OR & IC & OR & IC & OR & IC \\
\hline \multicolumn{9}{|l|}{ Sexo } \\
\hline Feminino & 1,00 & - & 1,00 & - & 1,00 & - & 1,00 & - \\
\hline Masculino & 1,73 & $(1,58-1,89)$ & 2,00 & $(1,82-2,21)$ & 1,15 & $(0,97-1,36)$ & 1,02 & $(0,85-1,24)$ \\
\hline \multicolumn{9}{|l|}{ Faixa-etária } \\
\hline 60 ou mais & 1,00 & - & 1,00 & - & 1,00 & - & 1,00 & - \\
\hline $40-59$ & 1,56 & $(1,30-1,87)$ & 1,26 & $(1,05-1,51)$ & 1,43 & $(1,19-1,72)$ & 1,23 & $(0,98-1,54)$ \\
\hline $20-39$ & 2,79 & $(2,34-3,33)$ & 1,81 & $(1,50-2,19)$ & 1,51 & $(1,19-1,92)$ & 1,14 & $(0,85-1,52)$ \\
\hline \multicolumn{9}{|l|}{ Raça/Cor de pele } \\
\hline Preta & 1,00 & - & 1,00 & - & 1,00 & - & 1,00 & - \\
\hline Parda & 0,99 & $(0,86-1,15)$ & 0,94 & $(0,80-1,09)$ & 1,35 & $(1,00-1,83)$ & 1,35 & $(0,95-1,93)$ \\
\hline Branca & 1,13 & $(0,98-1,32)$ & 0,89 & $(0,76-1,05)$ & 1,45 & $(1,07-1,95)$ & 1,15 & $(0,82-1,62)$ \\
\hline \multicolumn{9}{|l|}{ Estado civil } \\
\hline Solteiro(a) & 1,00 & - & 1,00 & - & 1,00 & - & 1,00 & - \\
\hline Separado/divorciado/viúvo(a) & 0,61 & $(0,52-0,71)$ & 0,97 & $(0,82-1,14)$ & 0,91 & $(0,72-1,15)$ & 1,03 & $(0,77-1,36)$ \\
\hline Casado(a) & 0,74 & $(0,68-0,81)$ & 0,85 & $(0,77-0,93)$ & 1,17 & $(0,95-1,44)$ & 1,04 & $(0,82-1,31)$ \\
\hline \multicolumn{9}{|l|}{ Escolaridade } \\
\hline Sem instrução & 1,00 & - & 1,00 & - & 1,00 & - & 1,00 & - \\
\hline Até fundamental completo & 1,74 & $(1,43-2,14)$ & 1,52 & $(1,26-1,85)$ & 1,58 & $(1,24-2,03)$ & 1,28 & $(0,98-1,66)$ \\
\hline Até médio completo & 3,82 & $(3,15-4,62)$ & 2,83 & $(2,33-3,44)$ & 2,71 & $(2,06-3,55)$ & 1,80 & $(1,34-2,42)$ \\
\hline Até superior completo & 6,22 & $(5,11-7,56)$ & 4,54 & $(3,71-5,57)$ & 4,54 & $(3,36-6,12)$ & 2,72 & $(1,94-3,79)$ \\
\hline \multicolumn{9}{|l|}{ Autopercepção da saúde } \\
\hline Negativa & 1,00 & - & 1,00 & - & 1,00 & - & 1,00 & - \\
\hline Positiva & 2,32 & $(2,07-2,61)$ & 1,48 & $(1,26-1,85)$ & 1,93 & $(1,61-2,32)$ & 1,49 & $(1,22-1,81)$ \\
\hline \multicolumn{9}{|l|}{ Espaço público para PAF } \\
\hline Não & 1,00 & - & 1,00 & - & 1,00 & - & 1,00 & - \\
\hline Sim & 2,11 & $(1,93-2,32)$ & 1,66 & $(1,50-1,84)$ & 2,04 & $(1,72-2,42)$ & 1,56 & $(1,28-1,91)$ \\
\hline \multicolumn{9}{|l|}{$\begin{array}{l}\text { Programas públicos que } \\
\text { estimulem a PAF }\end{array}$} \\
\hline Não & 1,00 & - & 1,00 & - & 1,00 & - & 1,00 & - \\
\hline Sim & 1,89 & $(1,70-2,09)$ & 1,49 & $(1,32-1,67)$ & 1,96 & $(1,65-2,34)$ & 1,52 & $(1,24-1,85)$ \\
\hline \multicolumn{9}{|l|}{$\begin{array}{l}\text { Recomendação de profissional } \\
\text { de saúde }\end{array}$} \\
\hline Não & - & - & - & - & 1,00 & - & 1,00 & - \\
\hline Sim & - & - & - & - & 3,99 & $(3,02-5,29)$ & 2,99 & $(2,22-4,03)$ \\
\hline
\end{tabular}

Ao avaliar a razão de chance para a PAFL suficiente entre pessoas com hipertensão arterial, análise ajustada apontou que indivíduos com até ensino médio completo e até superior completo apresentaram $80 \%$ e $172 \%$ maior chance de serem suficientemente ativos comparados a indivíduos sem instrução. Pessoas com autopercepção positiva da saúde, que relataram espaço público para a prática de atividade física e programas públicos para estimular a prática de atividade física apresentaram, respectivamente, $49 \%$, 56\% e $52 \%$ maior chance de serem suficientemente ativos. Indivíduos hipertensos que foram recomendados por profissional de saúde a praticar atividade física tiveram três vezes maior chance de serem ativos (Tabela 2).

Entre as pessoas com diabetes, análise ajustada mostrou variáveis com comportamento similar ao encontrado entre os indivíduos hipertensos, destacando que indivíduos com diabetes 
que receberam recomendação de profissional de saúde para a prática de atividade física tiveram 7,7 maior chance de serem suficientemente ativos. Aqueles com 40 a 59 anos foram $68 \%$ mais ativos que os mais idosos, em análise ajustada (Tabela 3).

Em análise ajustada, pessoas com hipercolesterolemia com até o ensino superior completo tiveram chance 3,1 vezes maior de serem suficientemente ativos no lazer comparados àqueles sem instrução. Indivíduos que relataram presença de espaço físico, programas públicos e recomendação de profissional de saúde para a prática de atividade física apresentaram, respectivamente, chance $52 \%, 65 \%$ e $124 \%$ maior de serem suficientemente ativos (Tabela 4).

\section{Discussão}

O presente estudo identificou em análise ajustada, nas três DCNT investigadas, maior chance de serem suficientemente ativos aqueles com autopercepção positiva da saúde, com maior escolaridade, que receberam recomendação de profissional de saúde para a PAFL e que relataramterem

Tabela 3. Razão de chance bruta e ajustada para PAFL suficiente em adultos ( $\geq 20$ anos) segundo presença de diabetes e fatores associados. PNS 2013.

\begin{tabular}{|c|c|c|c|c|}
\hline \multirow{3}{*}{ Variáveis } & \multicolumn{4}{|c|}{ Com diabetes } \\
\hline & \multicolumn{2}{|c|}{ Bruta } & \multicolumn{2}{|c|}{ Ajustada } \\
\hline & OR & IC & OR & IC \\
\hline \multicolumn{5}{|l|}{ Sexo } \\
\hline Feminino & 1,00 & - & 1,00 & - \\
\hline Masculino & 0,99 & $(0,71-1,37)$ & 0,78 & $(0,55-1,12)$ \\
\hline \multicolumn{5}{|l|}{ Faixa-etária } \\
\hline 60 ou mais & 1,00 & - & 1,00 & - \\
\hline $40-59$ & 1,99 & $(1,44-2,74)$ & 1,68 & $(1,13-2,50)$ \\
\hline $20-39$ & 2,20 & $(1,35-3,61)$ & 1,81 & $(0,97-3,37)$ \\
\hline \multicolumn{5}{|l|}{ Raça/Cor de pele } \\
\hline Preta & 1,00 & - & 1,00 & - \\
\hline Parda & 1,20 & $(0,72-1,98)$ & 1,04 & $(0,60-1,79)$ \\
\hline Branca & 1,61 & $(0,93-2,77)$ & 1,16 & $(0,64-2,08)$ \\
\hline \multicolumn{5}{|l|}{ Estado civil } \\
\hline Solteiro(a) & 1,00 & - & 1,00 & - \\
\hline Separado/divorciado/viúvo(a) & 0,98 & $(0,62-1,54)$ & 1,39 & $(0,79-2,45)$ \\
\hline Casado(a) & 1,26 & $(0,87-1,83)$ & 1,51 & $(0,96-2,37)$ \\
\hline \multicolumn{5}{|l|}{ Escolaridade } \\
\hline Sem instrução & 1,00 & - & 1,00 & - \\
\hline Até fundamental completo & 1,54 & $(1,00-2,35)$ & 0,95 & $(0,60-1,50)$ \\
\hline Até médio completo & 3,62 & $(2,20-5,97)$ & 1,96 & $(1,13-3,42)$ \\
\hline Até superior completo & 3,92 & $(2,25-6,83)$ & 2,10 & $(1,15-3,86)$ \\
\hline \multicolumn{5}{|l|}{ Autopercepção da saúde } \\
\hline Negativa & 1,00 & - & 1,00 & - \\
\hline Positiva & 1,90 & $(1,38-2,63)$ & 1,59 & $(1,11-2.26)$ \\
\hline \multicolumn{5}{|l|}{ Espaço público para PAF } \\
\hline Não & 1,00 & - & 1,00 & - \\
\hline Sim & 1,65 & $(1,18-2,32)$ & 1,46 & $(1,00-2,12)$ \\
\hline \multicolumn{5}{|c|}{ Programas públicos que estimulem a PAF } \\
\hline Não & 1,00 & - & 1,00 & - \\
\hline Sim & 1,81 & $(1,22-2,68)$ & 1,80 & $(1,16-2,78)$ \\
\hline \multicolumn{5}{|c|}{ Recomendação de profissional de saúde } \\
\hline Não & 1,00 & - & 1,00 & - \\
\hline Sim & 11,05 & $(6,32-19,33)$ & 7,71 & $(4,24-14,02)$ \\
\hline
\end{tabular}

Fonte: IBGE $^{32}$. 
Tabela 4. Razão de chance bruta e ajustada para PAFL suficiente em adultos ( $\geq 20$ anos) segundo presença de hipercolesterolemia e fatores associados. PNS 2013.

\begin{tabular}{|c|c|c|c|c|}
\hline \multirow{3}{*}{ Variáveis } & \multicolumn{4}{|c|}{ Com hipercolesterolemia } \\
\hline & \multicolumn{2}{|c|}{ Bruta } & \multicolumn{2}{|c|}{ Ajustada } \\
\hline & OR & IC & OR & IC \\
\hline \multicolumn{5}{|l|}{ Sexo } \\
\hline Feminino & 1,00 & - & 1,00 & - \\
\hline Masculino & 1,13 & $(0,91-1,41)$ & 1,01 & $(0,81-1,28)$ \\
\hline \multicolumn{5}{|l|}{ Faixa-etária } \\
\hline 60 ou mais & 1,00 & - & 1,00 & - \\
\hline $40-59$ & 1,48 & $(1,19-1,84)$ & 1,17 & $(0,92-1,49)$ \\
\hline $20-39$ & 2,03 & $(1,51-2,75)$ & 1,19 & $(0,82-1,74)$ \\
\hline \multicolumn{5}{|l|}{ Raça/Cor de pele } \\
\hline Preta & 1,00 & - & 1,00 & - \\
\hline Parda & 1,53 & $(1,01-2,33)$ & 1,41 & $(0,90-2,22)$ \\
\hline Branca & 1,66 & $(1,11-2,48)$ & 1,19 & $(0,77-1,83)$ \\
\hline \multicolumn{5}{|l|}{ Estado civil } \\
\hline Solteiro(a) & 1,00 & - & 1,00 & - \\
\hline Separado/divorciado/viúvo(a) & 0,79 & $(0,59-1,05)$ & 1,05 & $(0,77-1,44)$ \\
\hline Casado(a) & 0,94 & $(0,73-1,22)$ & 1,07 & $(0,81-1,41)$ \\
\hline \multicolumn{5}{|l|}{ Escolaridade } \\
\hline Sem instrução & 1,00 & - & 1,00 & - \\
\hline Até fundamental completo & 1,53 & $(1,09-2,15)$ & 1,26 & $(0,88-1,82)$ \\
\hline Até médio completo & 2,69 & $(1,89-3,82)$ & 1,70 & $(1,15-2,50)$ \\
\hline Até superior completo & 5,65 & $(3,92-8,14)$ & 3,13 & $(2,10-4,66)$ \\
\hline \multicolumn{5}{|l|}{ Autopercepção da saúde } \\
\hline Negativa & 1,00 & - & 1,00 & - \\
\hline Positiva & 2,51 & $(2,02-3,11)$ & 1,70 & $(1,33-2,18)$ \\
\hline \multicolumn{5}{|l|}{ Espaço público para PAF } \\
\hline Não & 1,00 & - & 1,00 & - \\
\hline Sim & 2,07 & $(1,68-2,55)$ & 1,52 & $(1,22-1,90)$ \\
\hline \multicolumn{5}{|c|}{ Programas públicos que estimulem a PAF } \\
\hline Não & 1,00 & - & 1,00 & - \\
\hline Sim & 2,00 & $(1,61-2,48)$ & 1,65 & $(1,31-2,07)$ \\
\hline \multicolumn{5}{|c|}{ Recomendação de profissional de saúde } \\
\hline Não & 1,00 & - & 1,00 & - \\
\hline Sim & 3,15 & $(1,97-5,06)$ & 2,24 & $(1,39-3,63)$ \\
\hline
\end{tabular}

espaço público próximo ao domicílio para a realização de atividade física ea presença no município de programas públicos que estimulem a PAF.

A relação inversa entre a PAF e a presença de DCNT é conhecida ${ }^{28}$, seja por meio de sua menor ocorrência nos mais suficientemente ativos ${ }^{9}$ ou ainda pela menor ocorrência de pessoas suficientemente ativas entre aqueles com alguma $\mathrm{DCNT}^{30,36}$. Em pessoas com as mesmas patologias, um estudo realizado com adultos brasileiros verificou maior prevalência da PAFL em homens, com maior escolaridade, maior renda, autorrela- to de saúde bom ou muito bom, que nunca fumaram, com menos morbidades, normopesos e com ambiente favorável para praticar atividade física $^{20}$. Na população geral brasileira a ocorrência da PAFL suficiente foi maior em homens, jovens, brancos, com ensino superior e residentes na área urbana ${ }^{17,19,23}$. Ter plano de saúde também esteve associado a maior PAFL, com prevalência de $43,9 \%$ em adultos residentes nas capitais brasileiras no ano de $2015^{8}$.

Entre as pessoas sem as patologias investigadas, o presente estudo identificou que os homens 
tiveram maior chance de serem fisicamente ativos em análise ajustada. No que se refere à diferença entre gêneros, inúmeros estudos apontam para maiores níveis de inatividade física ou atividade física insuficiente entre as mulheres ${ }^{16,17,19,23}$. Ao investigar a diferença de gênero na inatividade física em 142 países, estudo apontou para maior prevalência entre as mulheres (27\%) do que em homens $(20 \%)$, concluindo que uma redução de $4,8 \%$ desses valores entre as mulheres em todo o mundo alcançaria a meta da OMS de reduzir em $10 \%$ os níveis globais de inatividade física ${ }^{18}$. Neste estudo, a análise ajustada não mostrou diferença significativa na PAFL entre homens e mulheres com alguma das doenças investigadas. Em estudo com base no ELSA, realizado com adultos (35-74 anos) brasileiros com HAS, diabetes e hipercolesterolemia, Forechi et al..$^{20}$ encontraram prevalência de PAFL distinta entre os sexos. Uma hipótese deste estudo ter apresentado igualdade entre os sexos pode ser o fato de as mulheres adultas mais jovens com DCNT apresentarem níveis de PAF mais elevados, aproximando a ocorrência entre os sexos. Ainda assim, ressalta-se que as análises consideraram ajuste por gênero.

A escolaridade foi fortemente associada a maior chance de PAFL nas três patologias, mesmo resultado encontrado por Forechi et al. ${ }^{20}$. Estudo de Lima-Costa ${ }^{24}$ mostrou que adultos e idosos com o ensino médio concluído apresentaram maior prevalência de serem fisicamente ativos comparado àqueles que não o haviam concluído. Entre as hipóteses explicativas desta associação é o fato de a escolaridade estar relacionada a melhores condições gerais de vida, melhores salários, empregos, capacidade de compra de serviços e convívio em ambientes mais propícios para a PAF, além da melhor compreensão dos benefícios da PAF à saúde ${ }^{37,38}$. Neste sentido, um estudo investigou a associação da posição socioeconômica no curso da vida e da mobilidade social intrageracional com comportamentos relacionados à saúde e identificou que as pessoas que decresceram sua condição social da infância para a vida adulta, ou ascenderam até a classe média, tiveram maiores chances de serem fisicamente inativos, comparado àqueles que pertenciam a classe alta na infância e permaneceram nesta categoria na vida adulta ${ }^{39}$. Phelan et al. ${ }^{40}$, ao discutirem as condições sociais como causas fundamentais das desigualdades na saúde, destacam a importância de formas mais equânimes de tratamento e promoção de saúde, visto que aqueles que possuem melhores condições socioeconômicas normalmente recebem os serviços de melhor qualidade. Esta iniquidade na prestação de serviços é reforçada em estudo que mostra que aqueles com menor renda tiveram menor oferta de serviços de saúde, incluindo exames essenciais, quando comparado aos mais $\operatorname{ricos}^{41}$. O presente estudo, assim, vai ao encontro de outros que identificaram associação positiva entre a PAFL e a escolaridade ${ }^{17,19,23}$.

Lima-Costa et al. ${ }^{42}$ definem autopercepção do estado de saúde como uma estrutura multidimensional, que compreende aspectos socioeconômicos, a rede de apoio, as condições de saúde e o acesso e uso dos serviços de saúde. Estudo de Bezerra et al. ${ }^{43}$ investigou a autoavaliação de saúde no estado do Acre e mostrou que as maiores prevalências de percepção negativa de saúde estavam nos indivíduos fisicamente inativos e que declararam ter pelo menos um agravo à saúde. Uma possível justificativa para esta associação são os efeitos negativos das doenças crônicas sobre a saúde emocional ${ }^{44}$, aspecto que Stewart-Brown et al. ${ }^{45}$ colocam como a maior causa de incapacidade em adultos em idade ativa. A necessidade de deslocamento para o local da prática, a disposição do indivíduo e a presença das doenças também são aspectos $\operatorname{citados}^{46}$. Outros estudos confirmam esta associação ${ }^{22,28}$.

Os resultados deste estudo mostraram que ter recomendação de um profissional de saúde para praticar de atividade física aumentaram as chances de ser suficientemente ativo. A relação entre a recomendação profissional e a adesão à prática está bem documentada na literatura ${ }^{27,29}$. Ao investigar esta associação em idosos do Sul do Brasil, análise ajustada mostrou que aqueles que receberam recomendação tiveram chance $18 \%$ maior de ser suficientemente ativos ${ }^{21}$. $\mathrm{O}$ mesmo estudo mostrou, entre seis categorias profissionais, que $80 \%$ das recomendações para a PAF eram realizadas pelo médico ${ }^{47}$. Lopes et al. ${ }^{48}$ atentam para a importância da participação de todos os profissionais de saúde durante o aconselhamento para maior efetividade e destacam a Atenção Primária à Saúde e o Programa Academia da Saúde como espaço oportuno para esta prática. Ainda assim, de acordo com Loch et al. ${ }^{49}$ as informações ao paciente por meio do aconselhamento e grupos de saúde são importantes, mas não suficientes para garantir processos consistentes de escolha comportamental, destacando a importância de que as pessoas tenham a possibilidade objetiva de executar aquilo que é saudável para si. Assim, um ambiente favorável à PAFL passa a ser fundamental.

Um achado importante do presente estudo foi justamente a relevância de espaços públicos e 
políticas para a promoção da atividade física. Estudo realizado em São Paulo mostrou que a presença de dois ou mais tipos de locais de lazer num raio de 500 metros da residência esteve associado a uma chance $65 \%$ maior de caminhar ${ }^{26}$. Ainda assim, ao avaliarem os programas públicos de atividade física no Brasil, Ferreira et al. ${ }^{50}$ encontraram como principal barreira para a PAF a falta de tempo $(41 \%)$, seguida da falta de interesse pelas atividades físicas ofertadas $(29,7 \%)$ e a distância até o local (12,7\%). Apenas 20\% dos entrevistados conheciam a existência de algum programa de estímulo à AF. No Canadá, um estudo que investigou políticas públicas de saúde voltadas para promoção de atividade física como forma de prevenir as DCNT apontou para políticas direcionadas ao ambiente construído como alternativa eficiente, destacando soluções para o transporte ativo, iluminação e ambientes seguros ${ }^{51}$. Estudo mostrou em capitais brasileiras que os espaços públicos aumentaram a PAFL, independente das diferenças regionais ${ }^{25}$, e os programas públicos de atividade física tiveram maior participação daqueles mais vulneráveis comparados aos mais $\operatorname{ricos}^{50}$. Estes argumentos sedimentam a importância do ambiente construído para a prática de atividade física, sobretudo como ferramenta de combate às desigualdades. Assim, visando elevar os níveis de atividade física da população, o Ministério da Saúde, alinhado com o Plano de Ações Estratégicas para o Enfrentamento das DCNT no Brasil 2011-20225 , lançou o Programa Academia da Saúde no ano de 2011, no intuito de superar as barreiras estruturais à atividade física e hábitos saudáveis, especialmente para populações vulneráveis ${ }^{52}$. Ademais, outras estratégias devem ser pensadas, sobretudo localmente, de forma a melhor atender as demandas neste sentido.

Uma das limitações do presente estudo é seu delineamento transversal, que não permite estabelecer relações de causalidade entre as variáveis. Um outro ponto é o autorreferenciamento das morbidades investigadas, permitindo o viés de memória dos respondentes, além de poder ser influenciado pela cognição, linguagem e escolaridade dos respondentes. Entretanto, o autorrelato é amplamente utilizado em estudos epidemiológicos, permitindo resultados com boa concordância ${ }^{53,54}$. Importante registrar que o autorrelato não contemplou a severidade da doença, que pode ter associação com o desfecho e demais variáveis investigadas. Outra limitação é um possível viés de seleção, visto que as pessoas que autorreferiram alguma DCNT são as que potencialmente utilizam mais os serviços de saúde, excluindo-se aqueles com a doença, mas sem o diagnóstico. A PNS não permite identificar na população geral os indivíduos que faziam uso de medicamentos específicos às DCNT avaliadas de forma a deduzir a presença de alguma patologia, possibilitando a subestimativa das pessoas com HAS, diabetes e/ou hipercolesterolemia. Por último, a possível associação da recomendação profissional com a PAFL suficiente pode representar algum efeito de confundimento residual. Contudo, os achados deste estudo permitem a compreensão do comportamento ativo em pessoas com DCNT bastante prevalentes no Brasil, contribuindo para construção de programas mais assertivos a esta população. Um ponto forte deste estudo é a utilização de uma amostra nacional e representativa, com alto tamanho amostral em cada uma da DCNT de exposição. A escassez de estudos ${ }^{20}$ que explorem o comportamento da PAF entre adultos com DCNT, sobretudo de forma estratificada para cada uma das três doenças investigadas no Brasil, tornam os achados deste estudo oportunos para o melhor entendimento deste problema de saúde pública.

Este estudo mostrou prevalência da PAFL distinta entre pessoas com cada uma das três DCNT investigadas, além de associação com intensidade diferente entre elas e as variáveis exploratórias. As maiores magnitudes de associação estiveram relacionadas à escolaridade e à recomendação para a PAF. O grande distanciamento entre aqueles sem instrução daqueles com mais estudos representa clara desigualdade, apontando para necessidade de estratégias que promovam equidade. Ser recomendado por profissional de saúde para a PAFL também esteve associado com maior chance de ser suficientemente ativo, sendo importante estratégias de capacitação dos profissionais de saúde para elevar a ocorrência das recomendações para pessoas com alguma DCNT. Outro aspecto importante é que deve haver investimento no desenho de ambientes construídos adequados e estimulantes à atividade física no lazer, além de políticas públicas municipais para a prática de atividade física. Ademais, se faz necessária a realização de mais estudos investigando a PAFL e variáveis associadas, sobretudo contemplando outros determinantes que podem afetar esta relação. O monitoramento dos padrões da PAFL entre as pessoas com DCNT deve ser regularmente explorado no intuito de monitorar tendências nos padrões de atividade física, promovendo políticas mais eficazes. 


\section{Colaboradores}

PSC Silva trabalhou na concepção do manuscrito, análise dos dados e redação final. AF Boing trabalhou no delineamento, revisão crítica e aprovação da versão a ser publicada.

\section{Referências}

1. World Health Organization (WHO). Saving lives, spending less - A strategic response to noncommunicable diseases [Internet]. 2018 [acessado 2019 jun 22]. Disponível em: https://apps.who.int/iris/bitstream/ handle/10665/272534/WHO-NMH-NVI-18.8-eng. pdf?ua $=1$.

2. Malta DC, Stopa SR, Szwarcwald CL, Gomes NL, Júnior JBS, Reis AAC. A vigilância e o monitoramento das principais doenças crônicas não transmissíveis no Brasil - Pesquisa Nacional de Saúde, 2013. Rev Bras Epidemiol 2015; 18(Supl. 2):3-16.

3. Ramires EKNM, Menezes RCE, Longo-Silva G, Santos TG, Marinho PM, Silveira JAC. Prevalência e Fatores Associados com a Śndrome Metabólica na População Adulta Brasileira: Pesquisa Nacional de Saúde - 2013. Arq Bras Cardiol 2018; 110(5):455-466.

4. Malta DC, Bernal RTI, Souza MFM, Szwarcwald CL, Lima MG, Barros MBA. Social inequalities in the prevalence of self-reported chronic non-communicable diseases in Brazil: national health survey 2013. Int J Equity Health 2016; 15(1):153.

5. Malta DC, Oliveira TP, Santos MAS, Andrade SSCA, Silva MMA, GT de Monitoramento do Plano de DCNT. Avanços do Plano de Ações Estratégicas para o Enfrentamento das Doenças Crônicas não Transmissíveis no Brasil, 2011-2015. Epidemiol Serv Saude 2016; 25(2):373-390.

6. Saint-Maurice PF, Coughlan D, Kelly SP, Keadle SK, Cook MB, Carlson SA. Association of Leisure-Time Physical Activity Across the Adult Course With AllCause and Cause- Specific Mortality. JAMA Network Open 2019; 2(3):e190355.

7. Silva DAS, Naghavi M, Duncan BB, Schmidt MI, Souza MFM, Malta DC. Physical inactivity as risk factor for mortality by diabetes mellitus in Brazil in 1990, 2006, and 2016. Diabetol Metab Syndr 2019; 11:23.

8. Malta DC, Bernal RTI, Vieira Neto E, Curci KA, Pasinato MTM, Lisbôa RM, Cachapuz RF, Coelho KSC. Tendências de fatores de risco e proteção de doenças crônicas não transmissíveis na população com planos de saúde no Brasil de 2008 a 2015. Rev Bras Epidemiol 2018; 21(Supl. 1):e180020.

9. Zangirolani LTO, Assumpção D, Medeiros MAT, Barros MBA. Hipertensão arterial autorreferida em adultos residentes em Campinas, São Paulo, Brasil: prevalência, fatores associados e práticas de controle em estudo de base populacional. Cien Saude Colet 2018; 23(4):1221-1232.

10. Faludi AA, Izar COM, Saraiva JFK, Chacra APM, Bianco HT, Afiune Neto A, Membros da Sociedade Brasileira de Cardiologia. Atualização da Diretriz Brasileira de Dislipidemia e Prevenção da Aterosclerose 2017. Arq Bras Cardiol 2017; 109(2 Supl. 1):1-76.

11. Malta DC, Bernal RTI, Andrade SSCA, Silva MMA, Velasquez-Melendez G. Prevalência e fatores associados com hipertensão arterial autorreferida em adultos brasileiros. Rev Saude Publica 2017; 51(Supl. 1):11s.

12. Thompson D, Walhin JP, Batterham AM, Stokes KA, Cooper AR, Andrews RC. Effect of diet or diet plus physical activity versus usual care on inflammatory markers in patients with newly diagnosed type 2 diabetes: the Early ACTivity in Diabetes (ACTID) randomized, controlled trial. J Am Heart Assoc 2014; 3(3):e000828. 
13. Andrews RC, Cooper AR, Montgomery AA, Norcross AJ., Peters TJ, Sharp DJ, Jackson N, Fitzsimons K, Bright J, Coulman K, England CY, Gorton J, McLenaghan A, Paxton E, Polet A, Thompson C, Dayan CM. Diet or diet plus physical activity usual care in patients with newly diagnosed type 2 diabetes: the Early ACTID randomised controlled trial. Lancet 2011; 9(378):19-39.

14. Malachias MVB, Souza WKSB, Plavnik FL, Rodrigues CIS, Brandão AA, Neves MFT, Membros de Sociedade Brasileira de Cardiologia. 7a Diretriz Brasileira de Hipertensão Arterial. Arq Bras Cardiol 2016; 107(3 Supl. 3):1-83.

15. Sociedade Brasileira de Diabetes (SBD). Diretrizes da Sociedade Brasileira de Diabetes 2017-2018. São Paulo: Editora Clannad; 2017.

16. Guthold R, Stevens GA, Roley LM, Bull FC. Worldwide trends in insufficient physical activity from 2001 to 2016: a pooled analysis of 358 population-based surveys with 1,9 million participants. Lancet 2018; 6(10):e1007-e1086.

17. Malta DC, Andrade SSCA, Stopa SR, Pereira CA, Szwarcwald CL, Silva Júnior JB, Reis AAC. Estilo de vida da população brasileira: resultados da Pesquisa Nacional de Saúde, 2013. Epidemiol. Serv Saude 2015; 24(2):217-226

18. Mielke GI, Hallal PC, Rodrigues GBA, Szwarcwald CL, Santos FV, Malta DC. Prática de atividade física e hábito de assistir à televisão entre adultos no Brasil: Pesquisa Nacional de Saúde 2013. Epidemiol Serv Saude 2015; 24(2):277-286.

19. Cruz MS, Bernal RTI, Claro RM. Tendência da prática de atividade física no lazer entre adultos no Brasil (2006-2016). Cad Saude Publica 2018; 34(10):e00114817.

20. Forechi L, Mill JG, Griep RH, Santos I, Pitanga F, Molina MCB. Adherence to physical activity in adults with chronic diseases: ELSA-Brasil. Rev Saude Publica 2018; 52:31.

21. Flores TR, Gomes AP, Soares ALG, Nunes BP, Assunção MCF, Gonçalves H, Bertoldi AD. Aconselhamento por profissionais de saúde e comportamentos saudáveis entre idosos: estudo de base populacional em Pelotas, sul do Brasil, 2014. Epidemiol Serv Saude 2018; 27(1):e201720112.

22. Alkerwi A, Schuh B, Sauvageot N, Zannad F, Olivier A, Guillaume M, Albert A, Larsson CA, NESCaV project group. Adherence to Physical Activity Recommendations and Its Associated Factors: An Interregional Population-Based Study. J Public Health Res 2015; 4(1):406

23. Souza CA, César CLG, Barros MBA, Carandina L, Goudbaum M, Marchioni DML, Fisberg RM. Prevalência de atividade física no lazer e fatores associados: estudos de base populacional em São Paulo, Brasil, 2008-2009. Cad Saude Publica 2013; 29(2):270-282.

24. Lima-Costa MF. A escolaridade afeta, igualmente, comportamentos prejudiciais à saúde de idosos e adultos mais jovens? - Inquérito de Saúde da Região Metropolitana de Belo Horizonte, Minas Gerais, Brasil. Epid Serv Saude 2004; 13(4):201-208.
25. Manta SW, Del Duca GF, Silva KS, Rech CR, Gomes RDS, Maximiliano GI, Malta DC. Is the availability of open public spaces associated with leisure-time physical activity in Brazilian adults? Health Promot Int 2020; 35(1):e51-e58.

26. Florindo AA, Barrozo LV, Cabral-Miranda W, Rodrigues EQ, Turrell G, Goldbaum M, Galvão Cesar CL, Giles-Corti B. Public open spaces and leisure-time walking in brazilian adults. Int J Environ Res Public Health 2017; 14(6):553.

27. Herghelegiu AM, Moser A, Prada GI, Born S, Wilhelm M, Stuck AE. Effects of health risk assessment and counselling on physical activity in older people: A pragmatic randomised trial. PLoS One 2017; 12(7): 0181371

28. Bauman AE, Reis RS, Sallis JF, Well JC, Loos RJ, Martin BW. Correlates of physical activity: why are some people physically active and others not? Lancet 2012; 380(9838):258-271.

29. Leung AYM, Chau PH, Leung ISH, Tse M, Wong PLC, Tam WM, Leung DYP. Motivating Diabetic and Hypertensive Patients to Engage in Regular Physical Activity: A Multi-Component Intervention Derived from the Concept of Photovoice. Int J Environ Res Public Health 2019; 16(70):e1219.

30. Malta DC, Bernal RTI, Iser BPM, Szwarcwald CL, Duncan CB, Schmidt MI. Fatores associados ao diabetes autorreferido segundo a Pesquisa Nacional de Saúde, 2013. Rev Saude Publica 2017; 51(Supl. 1):12s.

31. Bueno RB, Marucci MFN, Codogno JS, Roediger MA. Os custos da inatividade física no mundo: estudo de revisão. Cien Saude Colet 2016; 21(4):1001-1010.

32. Instituto Brasileiro de Geografia e Estatística (IBGE). Pesquisa Nacional de Saúde - 2013: ciclos de Vida Brasil e grandes regiões. Rio de Janeiro: IBGE; 2015.

33. World Health Organization (WHO). Global recommendations on physical activity for health. Geneva: WHO; 2010.

34. American College of Sports Medicine (ACSM). Guidelines for exercise testing and prescription. $10^{\mathrm{a}} \mathrm{ed}$. Philadelphia: Lippincott Williams \& Wilkins; 2018.

35. U.S. Department of Health and Human Services. Physical Activity Guidelines Advisory Committee. Physical Activity Guidelines Advisory Committee Scientific Report. Washington, D.C.: U.S. Departmentof Health and Human Services; 2018.

36. Souza NA, Vieira SA, Fonsêca PCA, Andreoli CS, Priore SE, Franceschini SCC. Dislipidemia familiar e fatores associados a alterações no perfil lipídico em crianças. Cien Saude Colet 2019; 24(1):323-332.

37. Camelo LV, Figueiredo RC, Oliveira-Campos M, Giatti L, Barreto SM. Comportamentos saudáveis e escolaridade no Brasil: tendência temporal de 2008 a 2013. Cien Saude Colet 2016; 21(4):1011-1021.

38. Nayga RM. Schooling, health knowledge, and obesity Applied Econom 2000; 32(7):815-832.

39. Faleiro JC, Giatti L, Barreto SM, Camelo LV, Griep RH, Guimarães JMN, Fonseca MJM, Chor D, Chagas MCA. Posição socioeconômica no curso de vida e comportamentos de risco relacionados à saúde: ELSA-Brasil. Cad Saude Publica 2017; 33(3):e00017916. 
40. Phelan JC, Link BG, Tehranifar P. Social conditions as fundamental causes of health inequalities: theory, evidence, and policy implications. J Health Soc Behav 2010; 51:s28-s41.

41. Campbell DJT, Ronksley PE, Manns BJ, Tonelli M, Sanmartin C, Weaver RG, Hennessy D, King-Shier $\mathrm{K}$, Campbell T, Hemmelgarn BR, Interdisciplinary Chronic Disease Collaboration. The Association of Income with Health Behavior Change and Disease Monitoring among Patients with Chronic Disease. PLoS One 2014; 9(4):e94007.

42. Lima-Costa MF, Firmo JOA, Uchôa E. A estrutura da auto-avaliação da saúde entre idosos: projeto Bambuí. Rev Saude Publica 2004; 38(6):827-834.

43. Bezerra PCL, Opitz SP, Koifman J, Muniz PT. Percepção de saúde e fatores associados em adultos: inquérito populacional em Rio Branco, Acre, Brasil, 20072008. Cad Saude Publica 2011; 27(12):2441-2451.

44. Souza GNP, Alves RJR, Souza LPS, Rosa AJ. Prevalência de sintomas depressivos e/ou ansiosos em pessoas com hipertensão arterial sistêmica e/ou diabetes mellitus. Rev Portug Enferm Saude Mental 2018; 20:43-50.

45. Stewart-Brown S, Layte R. Emotional health problems are the most important cause of disability in adults of working age: a study in for countries of the old Oxford region. J Epidemiol Community Health 1997; 51(6):672-675.

46. Romeiro-Lopes T, França-Graven AA, Agnolo CMD, Rocha-Brischiliari SC, Carvalho MDB, Pelloso SM. Fatores associados à inatividade física no lazer em município do Sul do Brasil. Rev Salud Publica 2014; 16(1):40-52.

47. Hafele B, Siqueira FV. Aconselhamento para atividade física e mudança de comportamento em Unidades Básicas de Saúde. Rev Bras Ativ Fis Saude 2016; 21(6):581-592.

48. Lopes ACS, Toledo MTT, Câmara AMCS, Menzel HJK, Santos LC. Condições de saúde e aconselhamento sobre alimentação e atividade física na Atenção Primária à Saúde de Belo Horizonte-MG. Epidemiol Serv Saude 2014; 23(3):475-486.
49. Loch MR, Dias DF, Castro ASR, Guerra PH. Controle remoto ou remoto controle? A economia comportamental e a promoção de comportamentos saudáveis. Rev Panam Salud Publica 2019; 43:e18.

50. Ferreira RW, Caputo EL, Hafele CA, Jerônimo JS, Florindo AA, Knuth AG, Silva MC. Acesso aos programas públicos de atividade física no Brasil: Pesquisa Nacional de Saúde, 2013. Cad Saude Publica 2019; 35(2):e00008618.

51. Mcgreck JA, Kongats K, Voyer C, Nykiforuk CIL. Healthy Public Policy Options to Promote Physical Activity for Chronic Disease Prevention: Understanding Canadian Policy Influencer and General Public Preferences. J Phys Activ Health 2019; 16(7):565-574.

52. Malta DC, Silva JB. Policies to promote physical activity in Brazil. Lancet 2012; 21(280):195-196.

53. Melo CL, Amaral TLM, Amaral CA, Vasconcellos MTL, Monteiro GTR. Acurácia da hipertensão arterial sistêmica autorreferida em adultos de Rio Branco, Acre. Epidemiol Serv Saude 2019; 28(2):1-12.

54. Haapanen N, Miilunpalo S, Pasanen M, Oja P, Vuori I. Agreement between questionnaire data and medical records of chronic diseases in middle-aged and elderly Finnish men and women. Am J Epidemiol 1997; 145(8):762-769.

Artigo apresentado em 23/02/2020

Aprovado em 08/09/2020

Versão final apresentada em 10/09/2020

Editores-chefes: Romeu Gomes, Antônio Augusto Moura da Silva 